\title{
100Gbps PCI-Express Readout for the LHCb Upgrade
}

Paolo Durante <paolo.durante@cern.ch> PH-LBC (LHCb Online) 


\section{Overview}

- PCle40 board architecture

- PCle DMA controller architecture

- PCle DMA results 


\section{LHCb experiment at CERN (in 2020)}

Hadron \& Electromagnetic Calorimeters ( $6 \mathrm{~Tb} / \mathrm{s})$

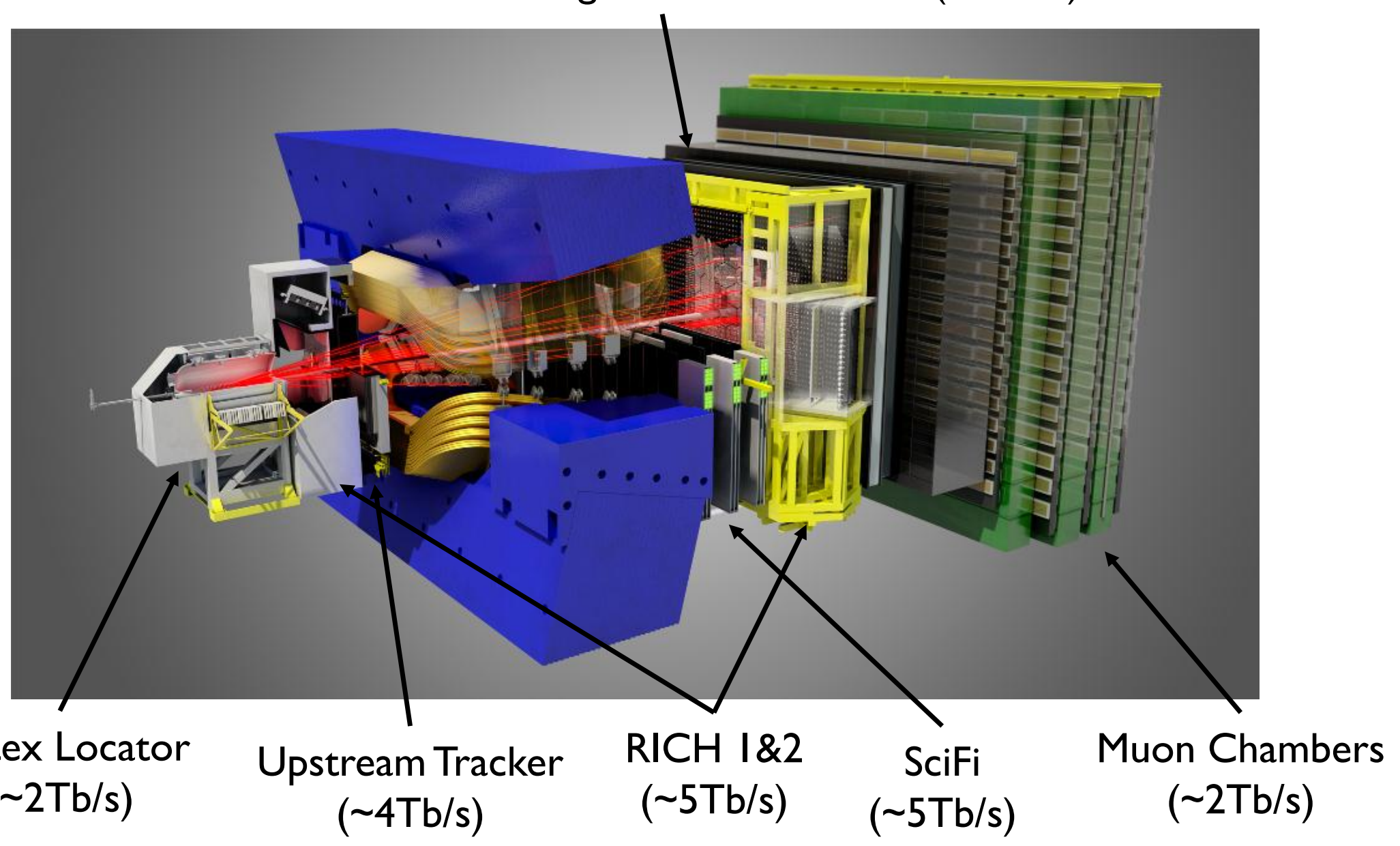




\section{High-level readout architecture}

\section{LHC THCP}
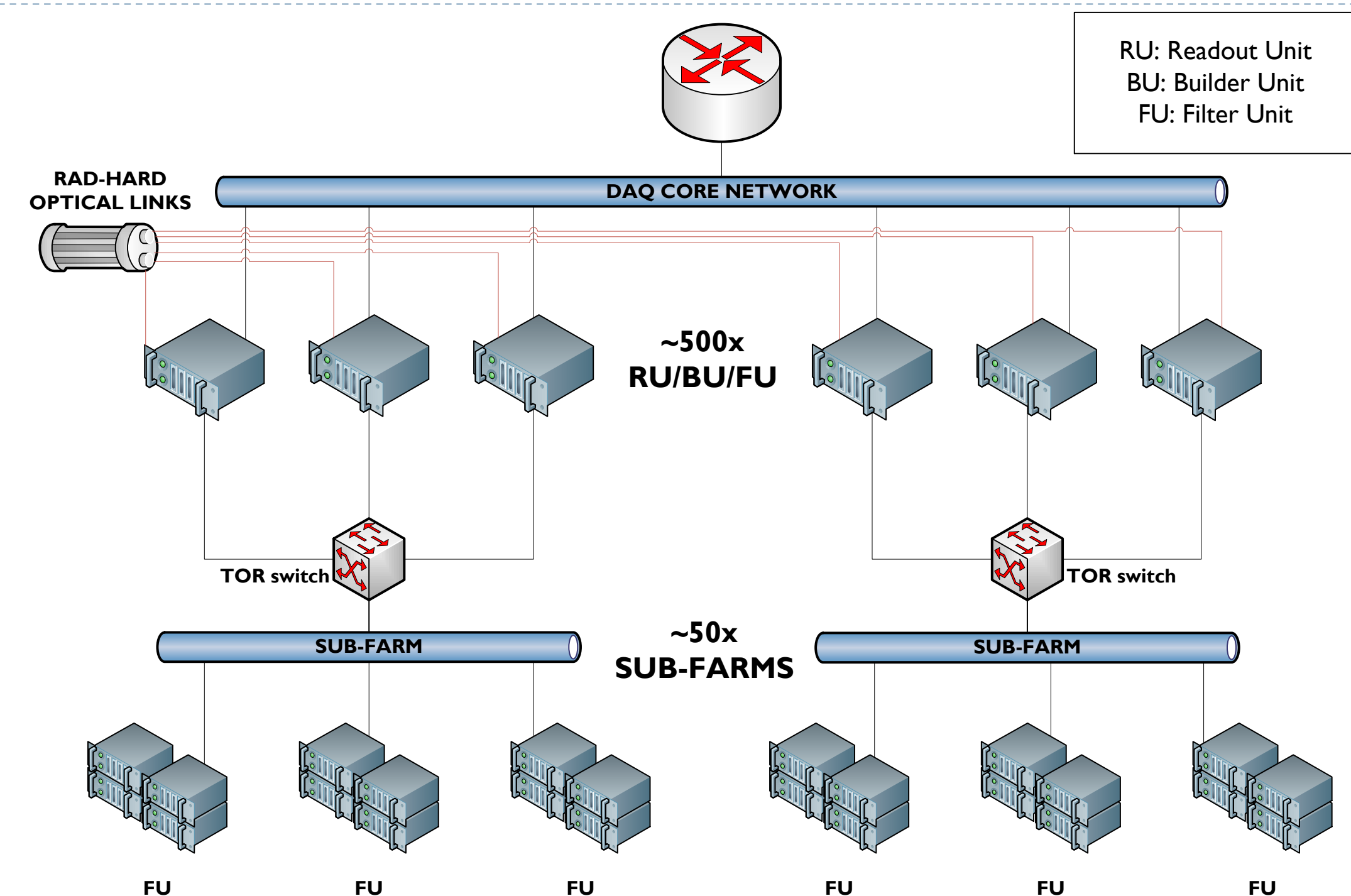

19th IEEE Real-Time Conference 20 I4 


\section{PCIe-40 architecture}

- 24 inputs in nominal DAQ configuration

- Up to 48 inputs for low occupancy sub-detectors

- UD to 48 bidirectional links for SOL40 configuration

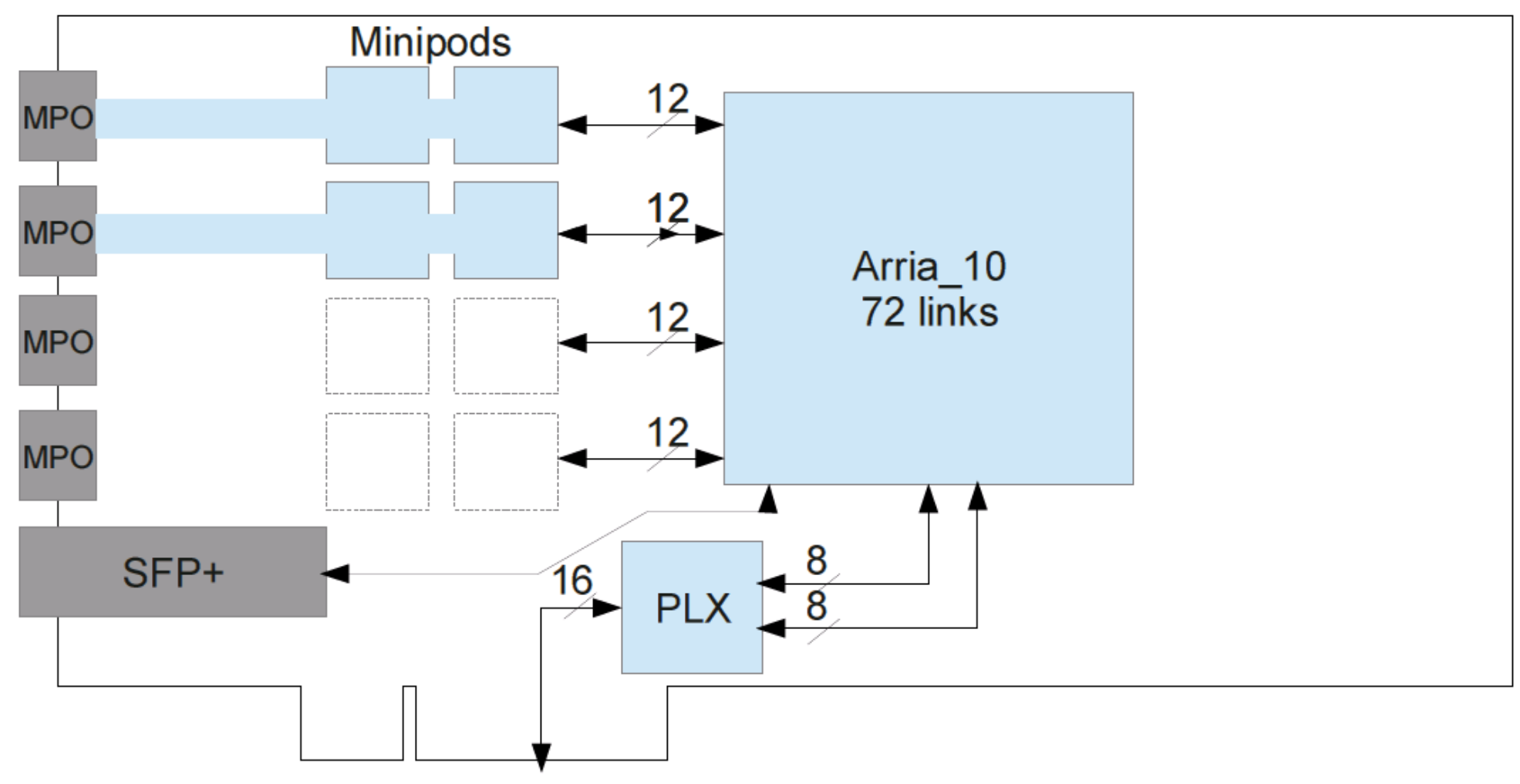




\section{PLX switch performance}

- Altera PCle IP only implements Gen3 x8

- Use two and bond them with a PCle switch

- nVIDIA does it with their dual GPGPUs, and it works!

- Sustained II2.8 Gbps observed downstream from switch

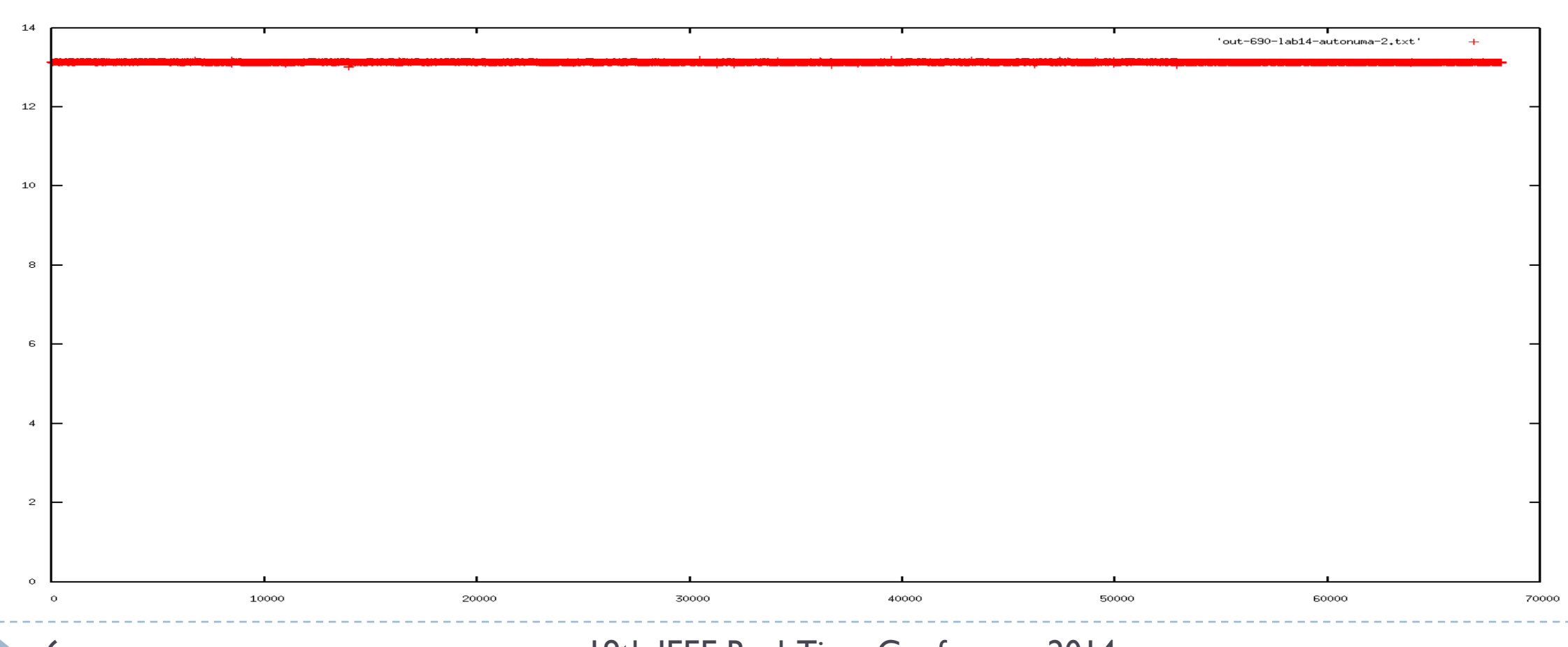




\section{PCIe DMA controller architecture}

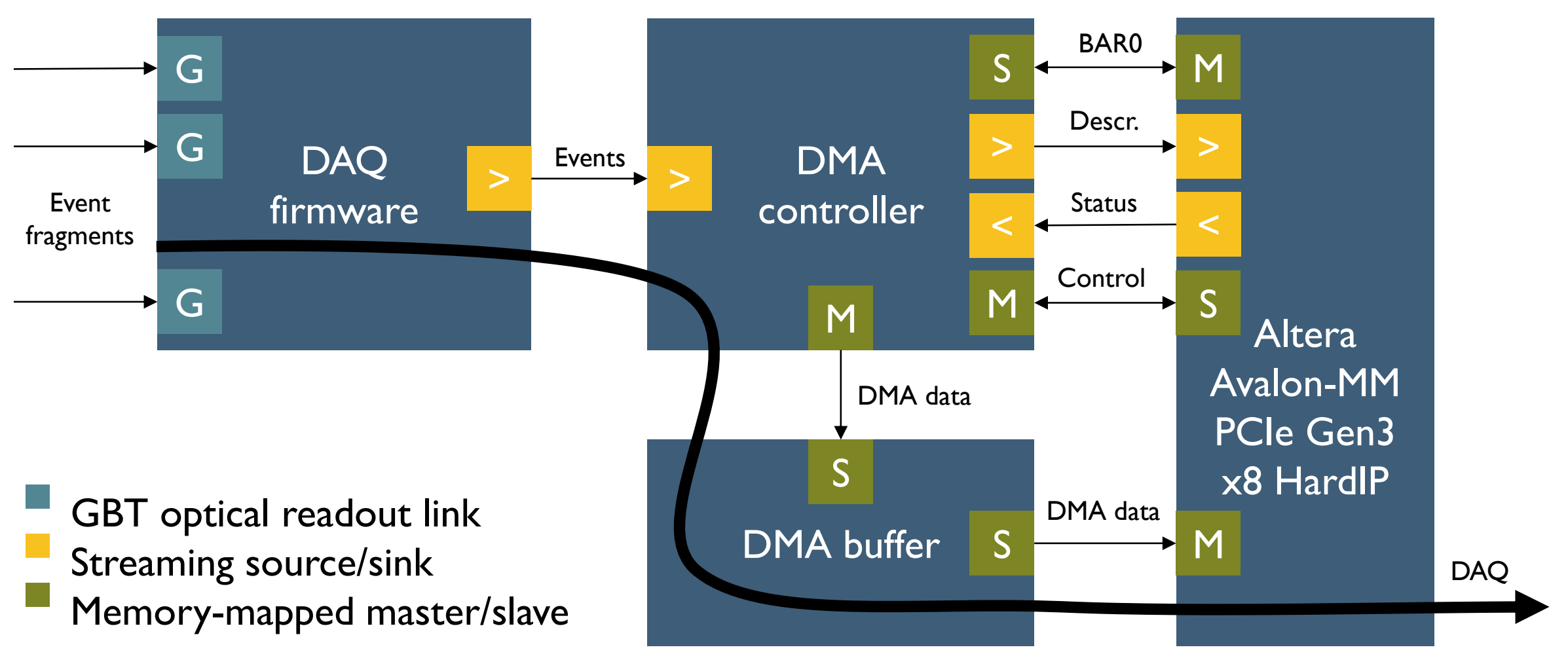

- Data path:256bits @ 250 MHz $\rightarrow 64$ Gbps 


\section{DMA descriptors}

- DMA engine tuning for Stratix $V$

1 16 descriptors

- Leaves headroom to absorb traffic bursts

> $4 \mathrm{KiB}$ per descriptor

- PCle MPS is $256 \mathrm{~B}$, no advantage in overloading DMA engine

- Matches MMU page size in event builder address space

\begin{tabular}{|c|c|c|c|c|c|c|c|}
\hline & \multicolumn{7}{|c|}{ Time } \\
\hline & SEND & WACK & WACK & WACK & WACK & FREE & D0 \\
\hline \multirow{4}{*}{$\begin{array}{l}\text { Write } \\
\text { order }\end{array}$} & FILL & FILL & SEND & SEND & WACK & WACK & DI \\
\hline & FREE & FREE & FILL & FILL & SEND & WACK & D2 \\
\hline & WACK & FREE & FREE & FREE & FILL & SEND & D3 \\
\hline & WACK & WACK & WACK & WACK & FREE & FILL & D4 \\
\hline
\end{tabular}




\section{DMA timing}

Acknowledgment of

DMA available for new descriptor previous descriptor

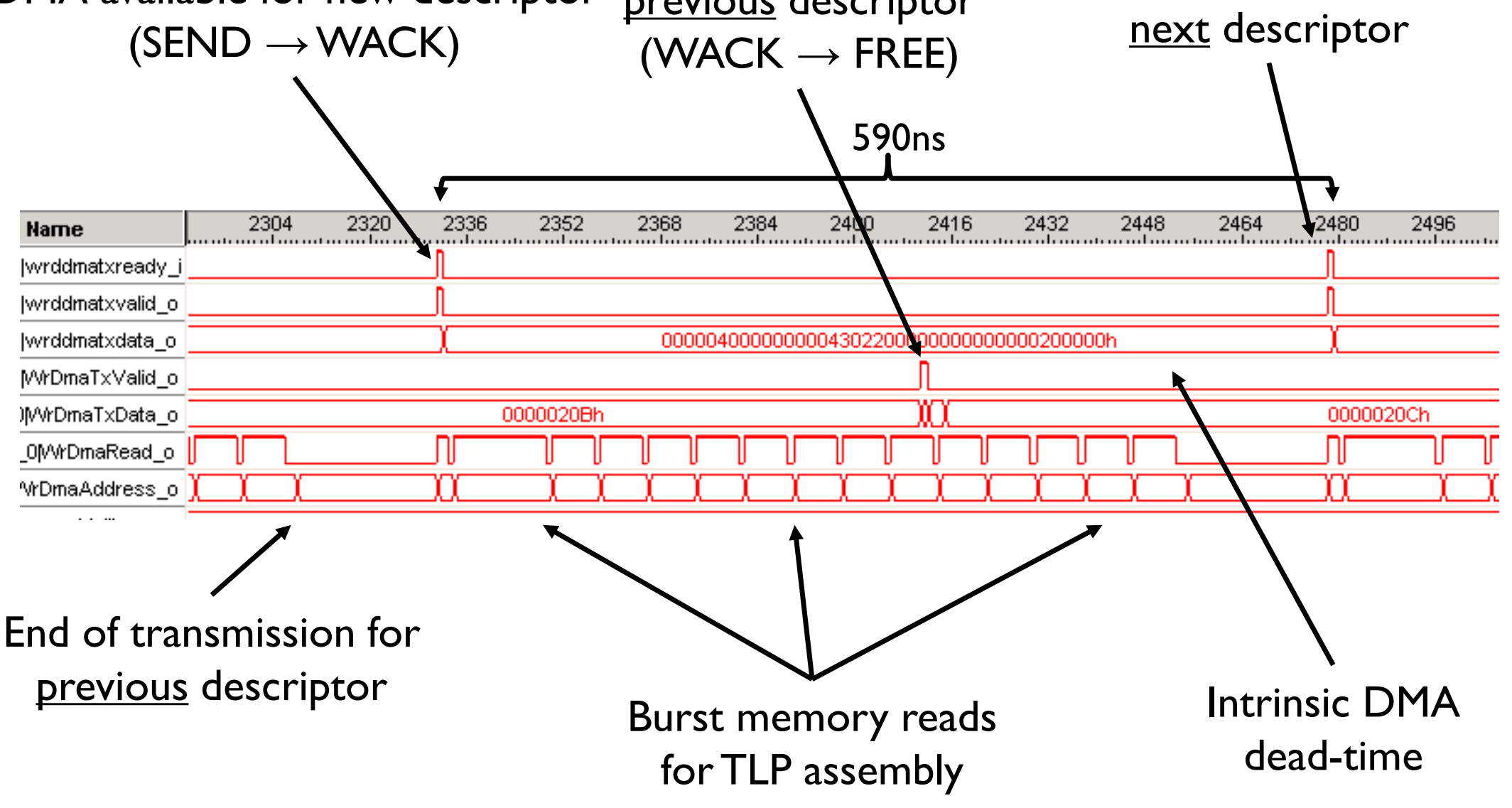




\section{DMA performance \& stability}

DMA rate sampling (12h run) 30000

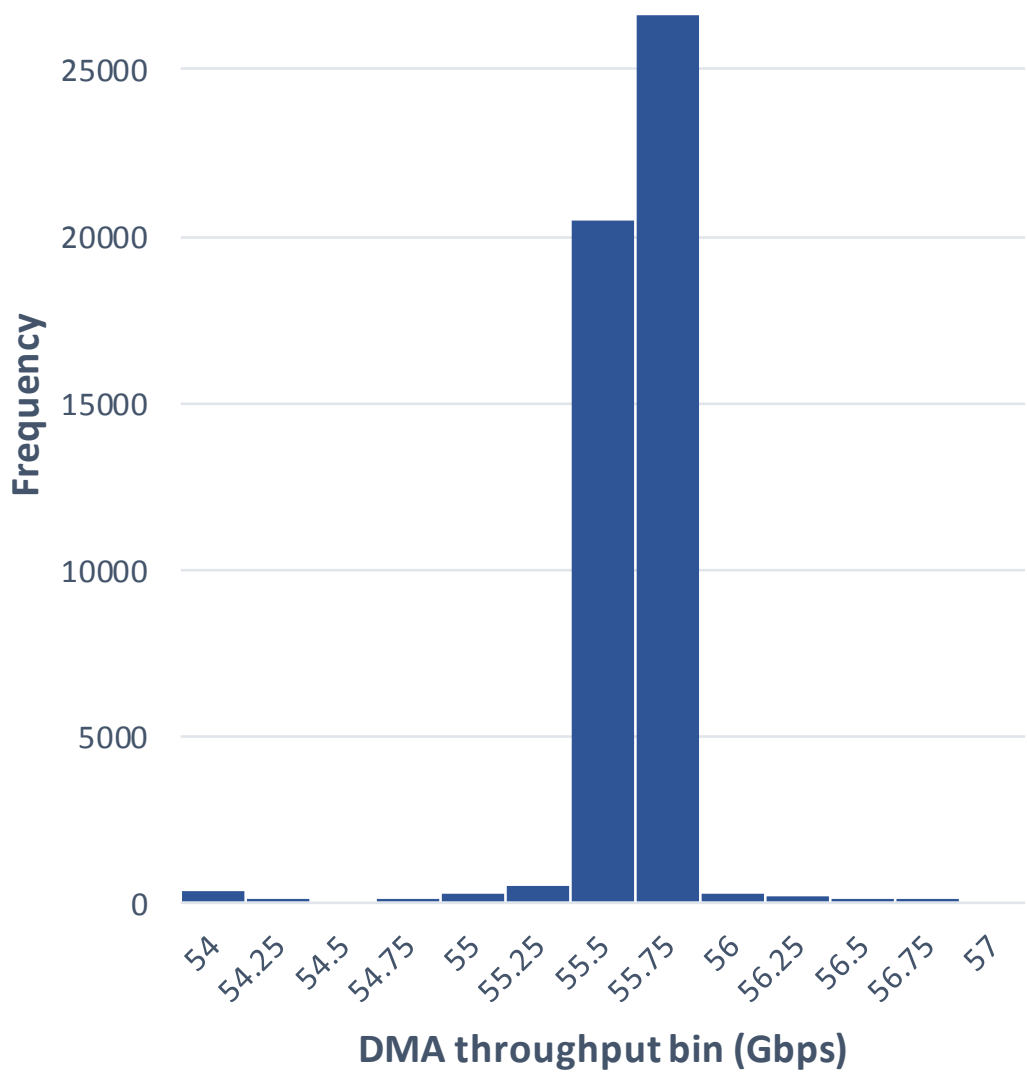

- Good performance

- 55.49 Gbps on average

- $96.9 \%$ of theoretical max

- Good stability

\pm 0.1 Gbps avg. deviation

- Driver and firmware stable over days of operation

- FPGA:Altera S5 (Bittware)

- CPU:Intel i7-3820 (Sandy) 


\section{Logic utilization}

MiniDAQ firmware synthesis results

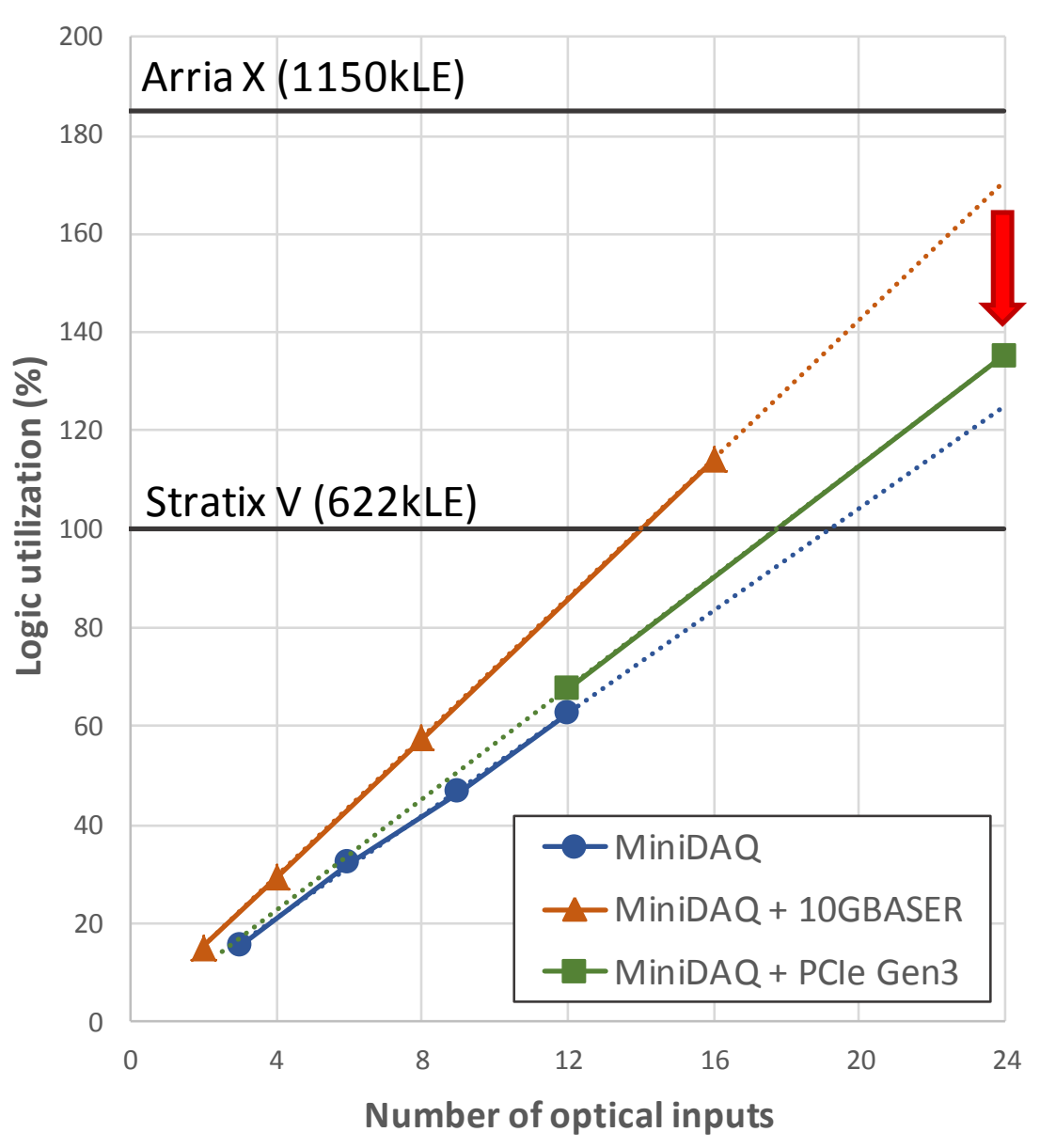

- Dynamic segment allocation minimizes onchip buffering

- $64 \mathrm{KiB}$ per instance

- Hardened IP minimizes reconfigurable logic resources

- $5 \%$ per instance

- MiniDAQ firmware is still heavy but constantly improving!

- Gen X devices are coming! 


\section{Control system interface}

- The PCle link will be used also by the control system

- Monitor and control internal state of readout board

- Instantiate an additional BAR to access firmware

- Is this going to create issues?

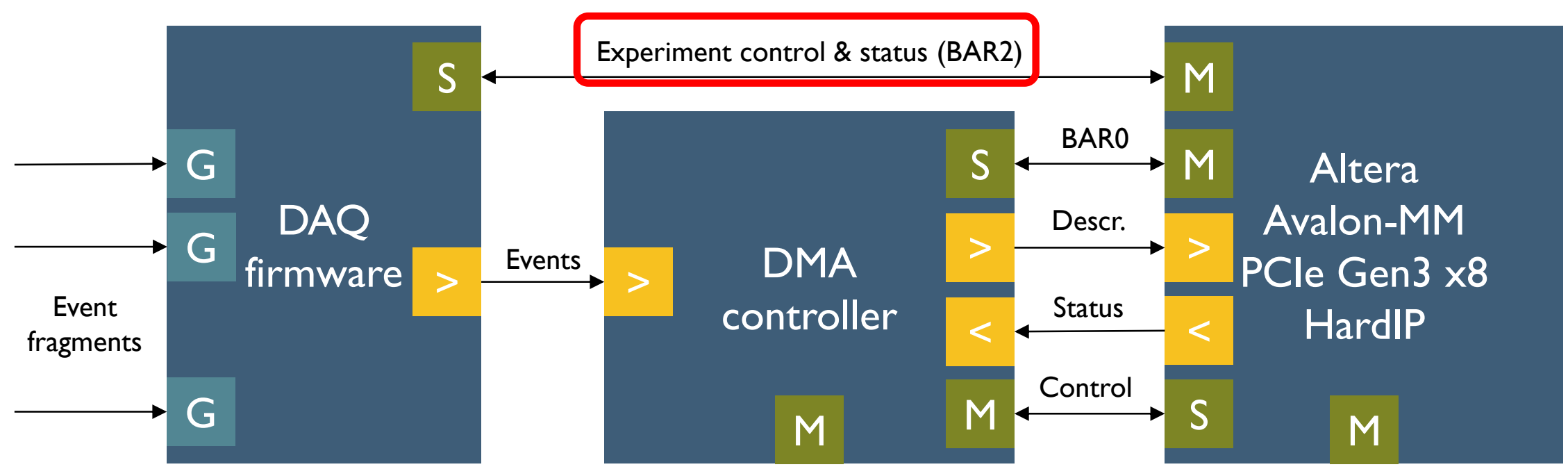




\section{Control system overhead}

- Implement control traffic emulator

- Evaluate different reads/writes ratio

- Performance still consistently over 54 Gbps!

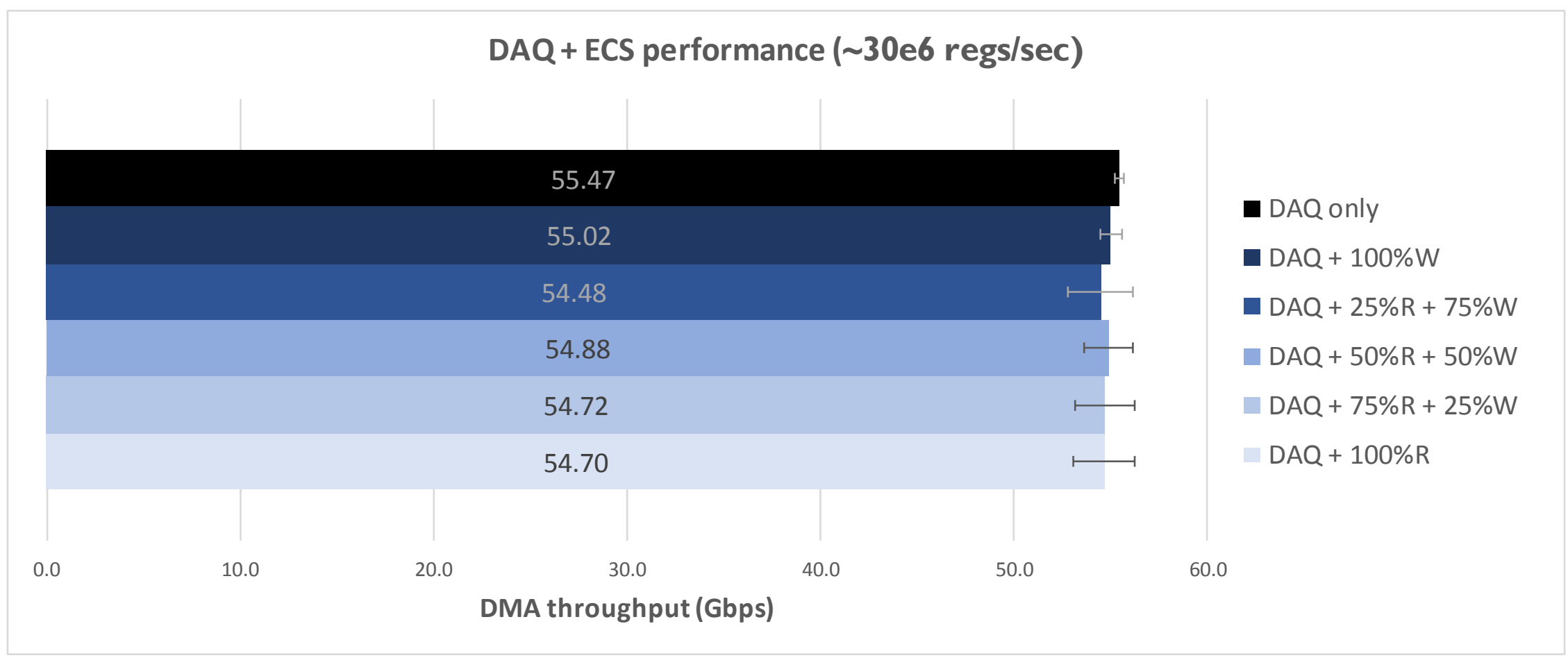




\section{Conclusions}

- DMA performance is close to maximum link capacity

- PCle output stage is more efficient wrt Ethernet readout

- Control \& status traffic over PCle does not disrupt DAQ

- DMA kernel driver and library are ready for integration in future event building software

- PCle switch tested on GPGPU but not yet on FPGA 


\section{Thank you! どうもありがとうございました}




\section{Modeling a PCI-e Gen3 link (1/2)}

- Protocol parameters

- Signaling speed: 8 GT/s

- Encoding overhead: I28b / I30b

- TLP header overhead: 20 Bytes

- Maximum payload size: 256 Bytes

- From the above we can readily obtain

- Packet efficiency: $92 \%$

- Symbol time: I.016ns / Byte

- TLP (256B+20B) transfer time: $~ 35 \mathrm{~ns}(\mathrm{x} 8) \sim 17.5 \mathrm{~ns}(\mathrm{x} / 6)$

- $\operatorname{DLLP}(8 \mathrm{~B})$ transfer time: $\sim$ Ins $(x 8) \sim 0.5 \mathrm{~ns}(\mathrm{x} \mid 6)$ 


\section{Modeling a PCI-e Gen3 link (2/2)}

- Descriptor size: $4 \mathrm{KiB}$

- TLPs per descriptor: $4096 / 256=16$

- DLLP traffic parameters

- Assume I ACK every 5 TLPs

- Assume I Flow Control update every 4TLPs

- Descriptor transfer time

- 8 lanes: $(\mid 6 \times 35 n s)+(|6 / 5 \times| n s)+(|6 / 4 \times| n s)=572.2 n s$

1 16 lanes: $(\mid 6 \times 17.5 n s)+(\mid 6 / 5 \times 0.5 n s)+(\mid 6 / 4 \times 0.5 n s)=283.6 n s$

- Theoretical Bandwidth

- 8 lanes: 4096Bytes / 572.2ns $=\mathbf{5 7 . 2 6 7}$ Gbps

- I6 lanes: 4096Bytes / 283.6ns = I I 5.54 Gbps 


\section{DMA buffering requirements}

- Events are committed to buffer in 32 Byte chunks

- Event header parsing + pass-through memory write

- FPGA buffer is divided in DMA sections

1 $\quad 4 \mathrm{KiB}$ sections $\rightarrow 88 \%$ bandwidth

$\downarrow \geq 8 \mathrm{KiB}$ sections $\rightarrow 87 \%$ bandwidth

- 16 sections $\rightarrow$ just $64 \mathrm{KiB}$ on the FPGA

- DMA does not need an external memory interface 


\section{DMA descriptor format}

- To every DMA section corresponds a descriptor

- Source (QSYS) address: 64bits

- Destination (Host) address: 64bits

- DMA length (dwords): I2bits

- Descriptor ID: 8bits

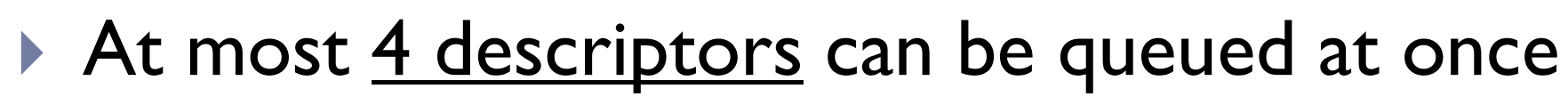

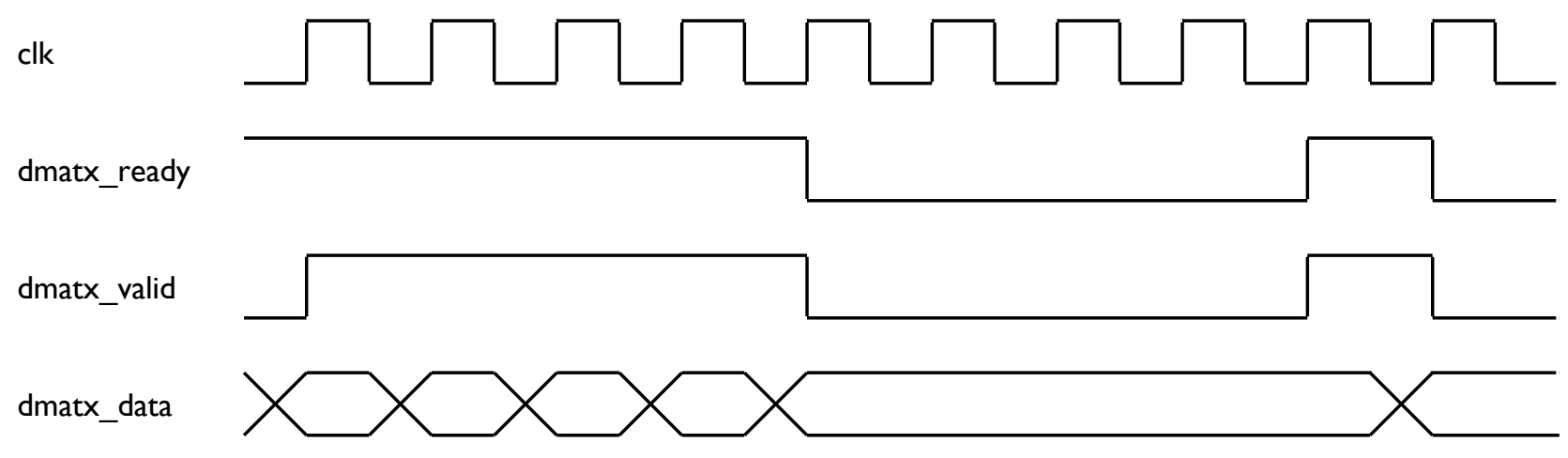




\section{MSI generation}

- Asynchronous signal to driver (and event builder)

- Triggered by Posted Write TLP at special host address

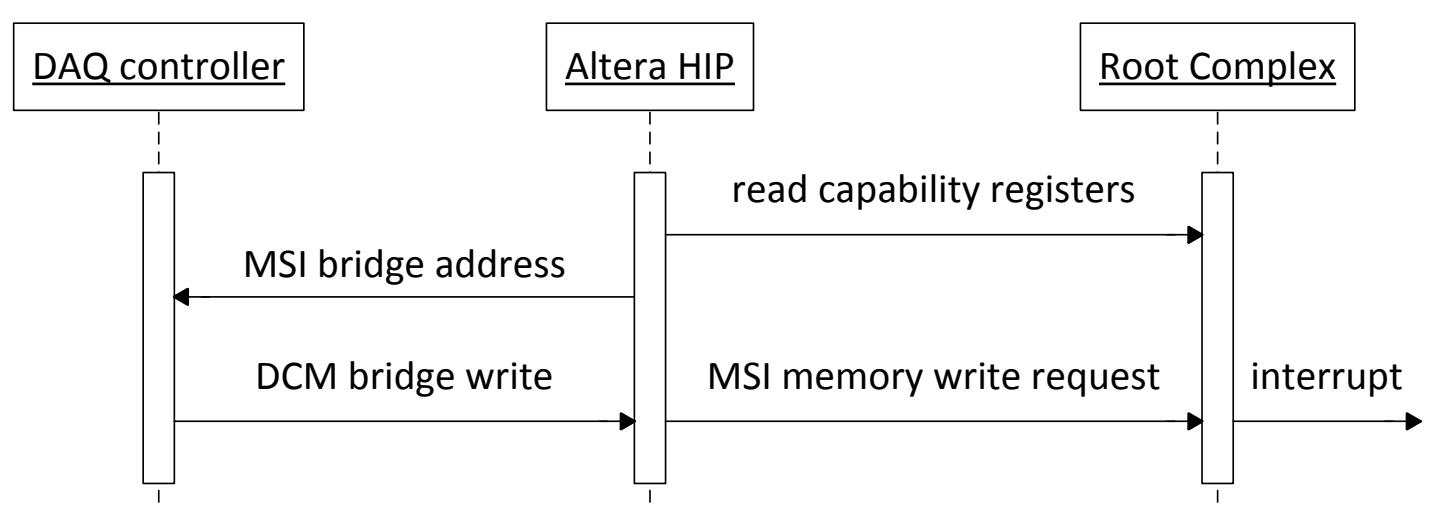

1 256 Gbps / $4 \mathrm{MiB}$

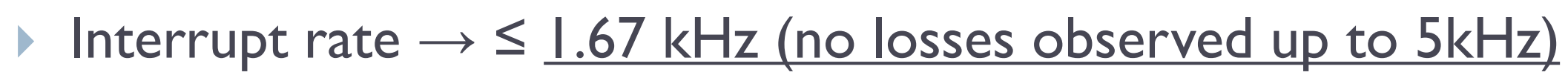




\section{Readout/Builder-Unit architecture}

Full events to farm

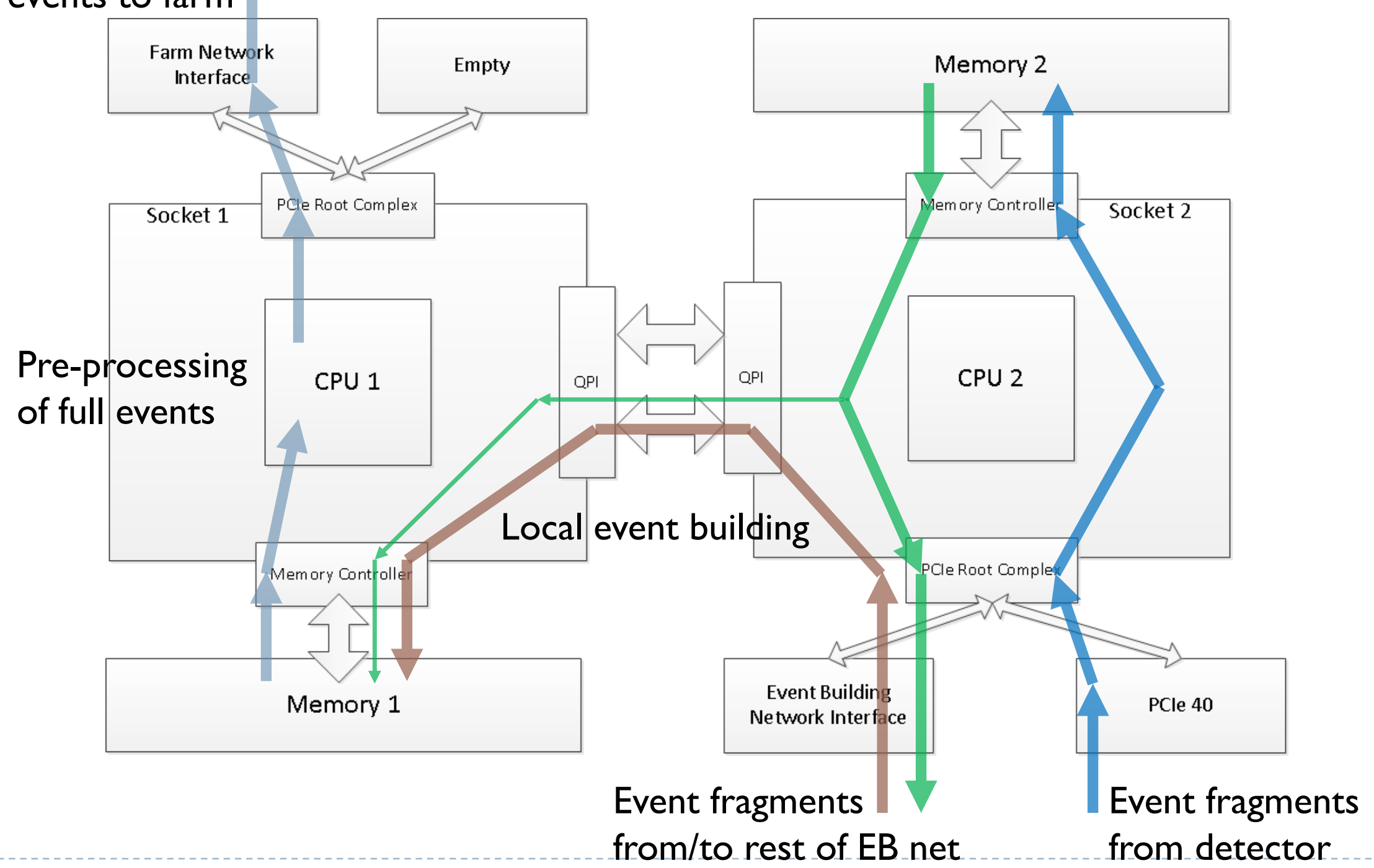

